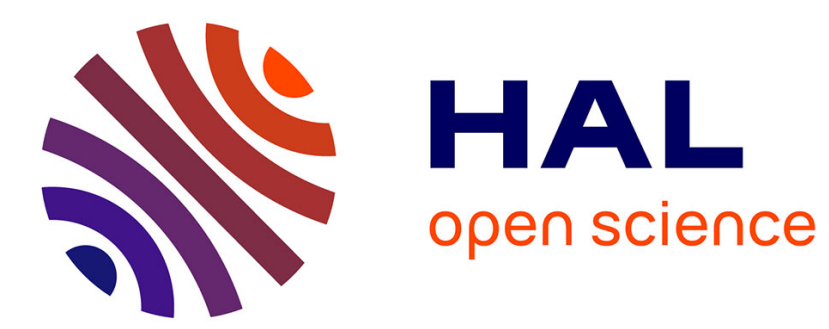

\title{
MOI ET L'AUTRE Le débat Giscard d'Estaing-Mitterrand
}

Dominique Labbé

\section{To cite this version:}

Dominique Labbé. MOI ET L'AUTRE Le débat Giscard d'Estaing-Mitterrand. Revue Française de Science Politique, 1981, 31 (5-6), pp.951-981. halshs-01719179

\section{HAL Id: halshs-01719179 \\ https://shs.hal.science/halshs-01719179}

Submitted on 28 Feb 2018

HAL is a multi-disciplinary open access archive for the deposit and dissemination of scientific research documents, whether they are published or not. The documents may come from teaching and research institutions in France or abroad, or from public or private research centers.
L'archive ouverte pluridisciplinaire HAL, est destinée au dépôt et à la diffusion de documents scientifiques de niveau recherche, publiés ou non, émanant des établissements d'enseignement et de recherche français ou étrangers, des laboratoires publics ou privés. 


\section{MOI ET L'AUTRE \\ Le débat Giscard d'Estaing-Mitterrand}

DOMINIQUE LABBÉ

Revue française de science politique. 31 (5-6), octobre-décembre 1981, p. 951-981.

La comparaison des interventions de MM. Giscard d'Estaing et Mitterrand, lors du débat qui les opposa le 5 mai 1981, révèle deux «énonciations » très différentes. L'analyse porte sur les pronoms, la structure actantielle, le temps et la «modalisation» du discours. Elle fait apparaître les présuppositions de deux adversaires et permet de comprendre quelles furent leurs stratégies de persuasion respectives.

\section{I and the Other Man. The Giscard d'Estaing-Mitterrand Debate}

Comparing what Mr. Giscard d'Estaing and Mr. Mitterrand said during their debate on 5 may 1981 reveals very different types of "enunciation". This analysis concerns the pronouns, actiantial structure, time and "modalisation" of their speech. It brings out the two opponents' presuppositions and clarifies their respective strategies of persuasion.

Manuscrit de l'auteur

Toute citation doit se faire à partir de la revue. 
Du débat télévisé qui, le 5 mai 1981, opposa MM. Giscard d'Estaing et Mitterrand, la postérité ne retiendra certainement que quelques «petites phrases» comme celles attribuant au candidat socialiste le «ministère de la parole» ou taxant l'ancien président d'«homme du passif». Ce n'est certes pas par hasard puisque les deux candidats ont organisé leur discours de telle sorte que ces slogans fassent mouche comme s'ils concentraient l'essentiel de leur pensée ou, tout au moins, de ce qu'ils tenaient à nous faire entendre. Mais en acceptant de les suivre sur ce terrain on risque de manquer les buts complexes, conscients ou non, que les candidats poursuivaient et qui se trouvaient présents derrière leurs propos, bien que difficilement décelables par l'auditeur. Ce phénomène continu qui se trouve à la source du discours, la linguistique le nomme «énonciation de la subjectivité» ou encore «présence du sujet parlant dans le texte $»^{1}$. Bien sûr, ici, il ne s'agit pas du caractère psychologique des candidats mais des stratégies de persuasion qu'ils avaient adoptées.

L'analyse de l'énonciation se heurte à deux difficultés. Tout d'abord, il est impossible d'établir en ce domaine des «normes de référence», ce qui limite le champ des études empiriques à des comparaisons comme celle qu'a menée L. Courdesses sur M. Thorez en 1936. Mais l'on sait que l'homme politique ne s'exprime pas de la même façon devant des militants ou dans une réunion de masse ${ }^{2}$. Il faudra donc que les deux orateurs comparés se soient trouvés dans des situations aussi semblables que possible afin que différences circonstancielles ne puissent être prises pour des traits pertinents. Or, justement, le débat télévisé écarte ces difficultés puisqu'il nous offre deux textes émis parallèlement dans des conditions formelles identiques. C'est pourquoi nous allons tenter, en analysant ce document, de montrer quelles furent les stratégies discursives de chacun des deux candidats $^{3}$.

\footnotetext{
${ }^{1}$ Benveniste (Emile), «L'homme dans la langue» et «L'appareil formel de l'enonciation», Problèmes de linguistique générale, Paris, Gallimard, 1966 et 1970. Dubois (Jean), « Enoncé et énonciation », Langages, 13, mars 1969. p. 100-110. Kerbrat-Orecchioni (Catherine), L'énonciation de la subjectivité dans le langage, Paris, A. Colin, 1981, etc. Nous ne discuterons pas les nombreux problèmes que pose cette théorie. Les concepts utilisés seront définis au moment de leur emploi.

${ }^{2}$ C'est le reproche que fait Louis Guespin au travail de Lucille Courdesses («Blum et Thorez en mai 1936». Langue française, 9 février 1971, p. 22-33 et «Problématiques des travaux sur le discours politique». Langages, 23, septembre 1971, p. 16).

${ }^{3}$ Nous avons analysé le compte rendu sténographique paru dans le journal Le Monde. La ponctuation rend donc le rythme oratoire des candidats sur lequel, par ailleurs, tout a été dit. Les références à la campagne de 1974 sont tirées de Cotteret (Jean-Marie) et al., 54774 mots pour convaincre, Paris. PUF, 1976, p. 79-115 et Charlot (Monica), Croizard (Isabelle), "Le discours télévisé des principaux candidats à la présidence de la République», Projet. 88. septembre-octobre 1974, p. 959-966.
} 
Il sera nécessaire de garder présentes à l'esprit quelques données caractéristiques. Au cours de la soirée, un peu plus de 21000 mots ont été prononcés par les deux interlocuteurs en 969 phrases. Comme à son habitude, M. Giscard d'Estaing a parlé plus vite, et de ce fait, le volume de ses interventions dépasse le volume de celles de M. Mitterrand de près de 2 000 mots en 100 phrases de plus. Nos statistiques sont donc établies en valeur relative afin de compenser cet écart. Enfin, il convient de rappeler que ce débat comprend quatre parties d'inégale longueur. Dans l'ordre, ont été traitées la politique intérieure qui représente $26 \%$ du débat, les questions économiques et sociales (43\%), la politique internationale (21\%) et. pour finir, la conclusion des deux candidats $(10 \%)$.

\section{LES ACTANTS DU DISCOURS ${ }^{4}$}

Poussant à l'extrême une caractéristique intrinsèque au langage, le discours politique met toujours en scène un petit drame, une certaine représentation de la réalité, qui comporte des personnages - abstraits ou concrets, êtres ou objets - que l'analyse linguistique nomme actants. Sans doute, le nom apparaît comme le moyen normal de désigner ces actants. Pourtant, il n'en sera rien, pour deux raisons. D'une part, la langue met à notre disposition un instrument commode, le « pronom », qui permet une économie appréciable. D'autre part et surtout, le discours politique, comme toute représentation, fait d'abord appel à la subjectivité : il propose à notre imagination des coordonnées qui ne naissent pas tant de la réalité que dans l'idéologie qui engendre ce discours. Or, de ce point de vue, le pronom offre un instrument remarquable sur lequel la lexicologie n'a rien pu nous apprendre de sérieux. C'est qu'il s'agit de «signes vides, non référentiels par rapport à la "réalité", toujours disponibles, et qui deviennent pleins dès qu'un locuteur les assume dans chaque instance de son discours» ${ }^{5}$. Autrement dit, à chaque utilisation, notre symbole se remplit d'un sens spécifique : non seulement le nous des communistes ne ressemble pas du tout au nous gaullien, mais chaque emploi se charge d'une nuance particulière qui lui vient du contexte lexical et de la situation dans laquelle il est prononcé. Pour autant, cela n'interdit pas leur recensement, car tout locuteur sélectionnera, dans l'infinité des acceptions possibles, les quelques traits spécifiques les mieux adaptés à la stratégie qu'il poursuit. C'est pourquoi, l'étude des pronoms utilisés lors du débat sera un bon révélateur des choix opérés par les

\footnotetext{
${ }^{4}$ Actant : terme employé par L. Tesnière pour désigner l'être ou l'objet qui participe d'une manière active ou passive au procès exprimé par le verbe, centre de la phrase.

${ }^{5}$ Benveniste (Emile), «La nature des pronoms», op. cit., p. 254.
} 
deux adversaires. De plus, les conditions d'une telle rencontre ont facilité notre tâche : il apparait, en effet, que la quasi-totalité des pronoms employés dans la soirée recouvre un petit nombre de sens que nous avons résumés dans l'encadré ci-après. Si les dénombrements opérés ont fait apparaître entre les deux locuteurs de très nettes différences, un trait s'impose cependant à l'observateur. Les deux adversaires structurent l'espace politique de la même manière, à l'aide de cinq catégories : le locuteur, son concurrent et leurs amis respectifs, les autres acteurs politiques, les Français et, enfin, l'impersonnel.

\section{La structure pronominale du débat}

Pour l'étude pronominale du débat, les décomptes suivants ont été opérés :

- Je. Désigne toujours le locuteur. Face à des énoncés du type «vous avez dit: "Je veux dissoudre"», on remplace le je par le nom de l'adversaire. Les autres formes du sujetpossessif (mon, ma...), réfléchi et complément d'objet (me) sont recensées à part.

- Vous. Pour la catégorie de l'autre, le tu ne se rencontre pas ici. Mais le pluriel de majesté (vous $\left.{ }_{1}\right)$ ne sera pas le seul usage du vous, il peut servir à désigner un groupe dans lequel le locuteur inclut son adversaire (vous 2 ). Par exemple, «dans le projet socialiste vous dites...». Enfin, il est utilisé pour s'adresser aux téléspectateurs (vous3). Le possessif est décompté à part et numéroté 1, 2 et 3 suivant la même nomenclature que ci-dessus.

- Nous. En dehors du pluriel de majesté qui ne se rencontre pas ici, hormis deux ou trois formes ambiguës chez M. Giscard d'Estaing. Le locuteur peut vouloir inclure son interlocuteur dans l'énoncé (récapitulatif: nous 1 ), ou d'autres, mais pas l'adversaire (par exemple son gouvernement, ses amis politiques.: nous 2 ) ou l'ensemble des auditeurs donc des Français qu'on appelle à adhérer ainsi à l'opinion émise (nous 3 ). Là encore le possessif est décompté à part suivant la même nomenclature.

- il, elle. La troisième personne peut être appliquée à l'interlocuteur $\left(i l_{1}\right)$ ou à une souscatégorie de $i l_{1}$ (son programme, son gouvernement, etc.). Il peut désigner une autre personne que le locuteur ne rattache pas à lui-même ou à son vis-à-vis $\left(i l_{2}\right)$. Il peut enfin s'agir de l'impersonnel neutre: il est vrai, il y a, il importe, etc. $\left.i l_{3}\right)$. L'attribut, les compléments, le réfléchi sont également comptés à part.

- Ils, elles. Peuvent désigner des amis ou des adversaires $\left(i l_{1}\right)$ des autres $\left(i l_{s_{2}}\right)$ ou encore les Français (ils 3 ). Même procédé que ci-dessus pour les autres formes pronominales de la troisième personne du pluriel.

- On. Certainement le cas le plus complexe. Il peut être inclusif (le locuteur se compte dedans). Ce sera par exemple un nous 1 ou un nous 2 inassumés («pour les prix agricoles, on a 
obtenu...», VGE) ou encore un nous $_{3}$ («on ne peut plus vous faire confiance», FM). Dans d'autres cas, il exclut implicitement le je et sert à dire «d'autres mais pas moi», avec une nuance péjorative («on se flatte beaucoup dans les propos officiels», FM). D'autres propositions implicites peuvent être suggérées grâce à on. Par exemple: «on ne peut pas défendre des théories de cette nature» (VGE) qui contient «il $l_{3}$ n'est pas permis de...», etc.

\section{Le locuteur}

Le poids du je apparaît considérable. Les deux candidats en usent beaucoup plus que de tout autre pronom, ce qui marque la personnalisation extrême à laquelle pousse l'élection présidentielle, et qui semble être assumée par les orateurs. Cependant, M. Mitterrand distance nettement M. Giscard d'Estaing sur ce point (29,5\% contre $21 \%$ o). La différence doit d'autant plus être notée qu'en 1974 elle était inverse. Le 5 mai 1981, contrairement à une habitude avérée chez lui, l'ancien président s'est un peu effacé de son propre discours, ce qui mérite d'être expliqué.

On comprendra aisément la différence entre «je veux lutter contre le chômage» et «il faut lutter contre le chômage». Si, au fond, les deux énoncés signifient la même chose, dans le premier cas, le sujet de l'énonciation est aussi celui de l'énoncé — on dira qu'il le prend en charge, qu'il l'assume - alors que, dans le second, le locuteur s'efface et pose son énoncé comme appartenant à la réalité extérieure, à l'ordre de la nécessité : on dira qu'il s'en distancie. C'est le principe du discours scientifique ou didactique d'où le je semble banni. Cet exemple permet de faire comprendre que l'intérêt d'une telle distinction provient de ce que cette distance semble s'établir entre le sujet et le monde, alors qu'en réalité «ce qui est communiqué à l'interlocuteur, ce n'est ni le sujet ni l'expérience mais le fait que ce qui est transmis est plus ou moins pris en charge» ${ }^{6}$. Sur cette courbe du plus ou moins assumé, M. Mitterrand se place incontestablement du côté du plus et M. Giscard d'Estaing vers le moins. Il semble marquer une distance plus grande par rapport à ce qu'il dit. Mais, surtout, il s'efface relativement, parle moins de lui. Ce phénomène nous livrera peu à peu la principale clef de ce débat.

Mais avant d'approfondir cette question, il convient de remarquer que le sujet, en plus $\mathrm{du}$ je, dispose d'une autre famille de signes pour s'incarner dans le discours: il s'agit de $m e$, moi et mon, ma, mes... Sur ce point, la différence entre les deux adversaires est encore plus

\footnotetext{
${ }^{6}$ Dubois (Jean), «Enoncé et énonciation», art. cité, p. 104.
} 
nette (Giscard d'Estaing, 5,5\%o contre 9,5\%o pour Mitterrand). Au total, l'ancien président semble plus porté sur le pronom de la première personne dans une proportion de quatre sur cinq manifestations du sujet. Au contraire, son adversaire socialiste utilise beaucoup plus nettement le possessif et le réfléchi (un pour quatre). En effet, l'emphase (moi...), très rare, est un peu plus utilisée par M. Giscard d'Estaing. Le possessif (mon programme, ma position, etc.), le réfléchi et le complément (je m'interroge, il me reproche...) ont en commun d'augmenter la distance : le locuteur devient l'objet ou le destinataire apparent de l'énoncé au lieu d'en être le sujet. On comprend dès lors qu'ils soient plus fréquents chez le candidat socialiste puisqu'ils lui permettent à la fois d'être le centre de son discours et de combattre l'effet produit par l'usage abondant de la première personne. Globalement donc, M. Giscard d'Estaing marque une plus grande distance par rapport à ce qu'il dit, mais assume mieux les énoncés explicitement consacrés à lui-même en se posant plus facilement en sujet de son énoncé.

En additionnant les occurrences de tous ces pronoms de la première personne, on constate que la différence se creuse encore entre les deux candidats. M. Mitterrand est en moyenne une fois et demie plus présent dans son discours que l'ancien président. Mais ce phénomène se manifeste inégalement (figure 1).

On voit dans ce graphique que la distance mise par les locuteurs entre leurs paroles et eux-mêmes varie beaucoup suivant le sujet abordé. M. Mitterrand semble assumer pleinement la première partie du débat (politique intérieure), mais prend ensuite une distance assez nette dans la partie concernant l'économie et surtout dans celle qui porte sur la politique internationale, ce qui peut tenir aux forces et aux faiblesses du candidat socialiste mais découler également de sa stratégie de persuasion qui nous permettra de comprendre pourquoi, curieusement, la distance reste aussi forte dans la conclusion du candidat socialiste. Au contraire, la politique intérieure et plus encore l'économie sont traitées de façon très distanciée par l'ancien président qui assume mieux la politique internationale et pleinement sa conclusion. On n'aura aucun mal à lire ici le bilan du septennat tel que l'établit, lui aussi, M. Giscard d'Estaing, malgré ses dénégations... Face à cette situation intérieure qu'il semble mal assumer, il a adopté une attitude particulière qui consiste, dans les deux premières parties, soit plus des deux tiers du débat, à parler plus de son adversaire que de lui-même (figure 1). 


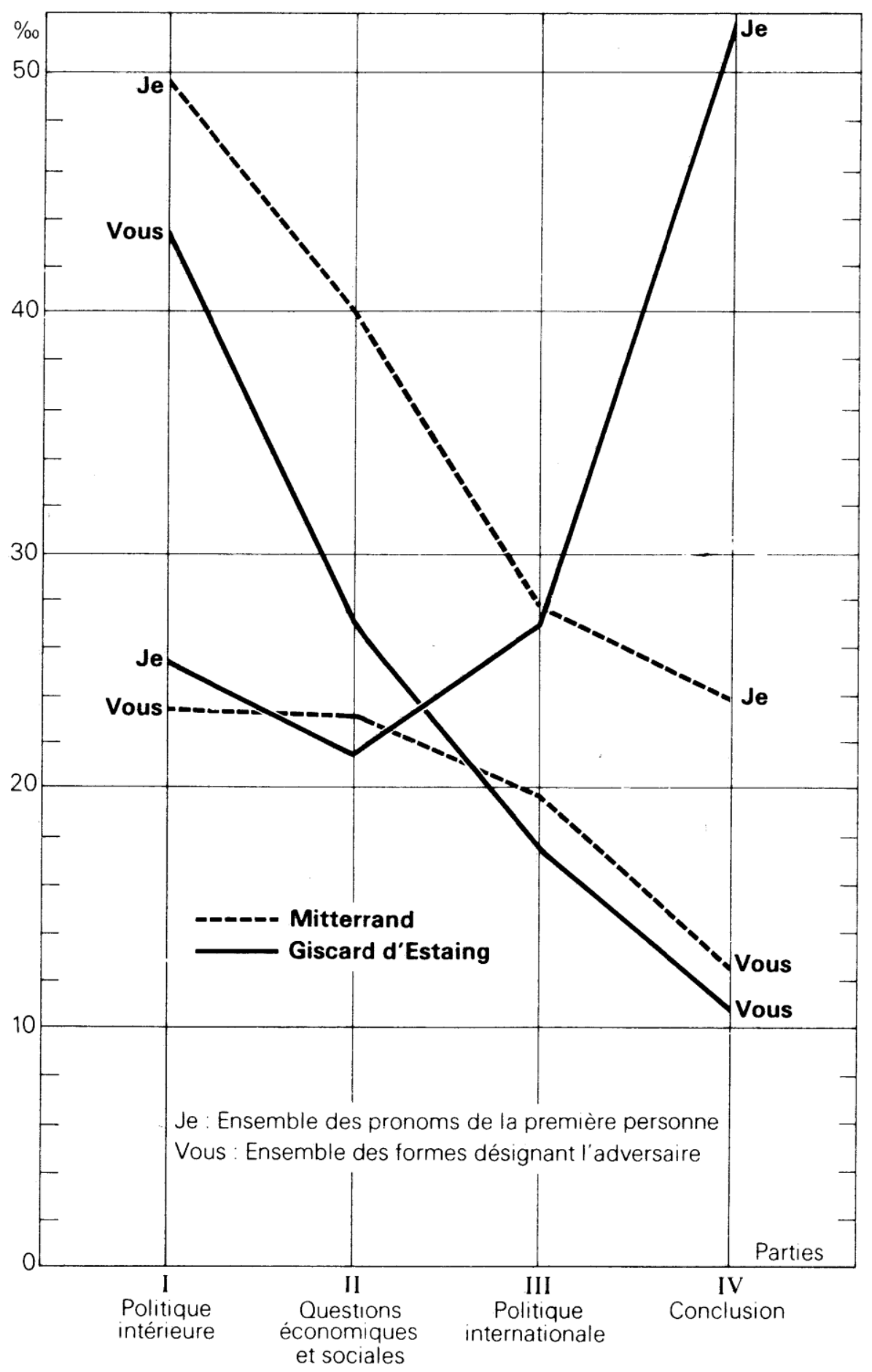

Figure I: Moi et l'autre*

* L'axe vertical indique la fréquence avec laquelle chacun des adversaires a utilisé les formes de la première personne ou celles désignant son adversaire. L'axe horizontal représente les parties du débat. Ainsi on voit que, dans la partie politique, Mitterrand a dit je (ou un dérivé) 50 fois en moyenne par tranche de mille mots: soit un sur vingt. Et, dans le même temps, il n'a désigné son adversaire que 22 fois. Mais, dans sa conclusion, le je a perdu la moitié de son poids. Giscard, lui, suit un mouvement à peu près inverse. Les points ont été reliés pour faciliter la lecture et figurer le déroulement du débat. 


\section{L'adversaire}

La loi d'un face-à-face réside dans le dialogue avec l'adversaire. D'où l'usage logique du vous 1 très fréquent chez les deux locuteurs. Cette relation entre le sujet et son interlocuteur peut être analysée à l'aide du concept de tension qui mesure la «volonté de communiquer» ${ }^{7}$. Ici, la tension ne désigne pas le désir de s'approprier l'autre, de gagner sa compréhension ou sa conviction, mais la détermination à l'affronter et, si possible, à le dominer. De ce point de vue, la détermination semble surtout être le fait de l'ex-président : il a utilisé en moyenne deux fois plus vous 1 que son adversaire, ce qui a conféré à son discours un caractère très tendu, au moins pour les deux premiers tiers du débat (et plus particulièrement au début). Comme en 1974, M. Giscard d'Estaing a choisi d'attaquer son adversaire plus qu'il ne plaide sa propre cause. Mais, alors que sept ans auparavant le candidat socialiste avait adopté une attitude sensiblement symétrique ${ }^{8}$, cette fois il s'est étendu davantage sur ses positions que sur le procès de son adversaire. Ce changement conféré au débat de 1981 un visage tout à fait neuf.

Donner à la polémique un tour plus ou moins agressif est évidemment affaire de tempérament, de style personnel, mais peut dépendre aussi de la conception qu'on se fait des attentes du public. Or de nombreux indices montrent que les deux candidats avaient, làdessus, des vues opposées dont on se souvient qu'elles se sont manifestées dans une polémique à propos de l'organisation même de ce débat. Par exemple, en dehors du $\operatorname{vous}_{1}$, il existe beaucoup d'autres manières de désigner l'interlocuteur: nous les résumons dans le tableau 1 où peut se lire une différence frappante dans les emplois.

\footnotetext{
${ }^{7}$ Ibid., p. 106.

${ }^{8}$ Cf. Cotteret (Jean-Marie) et al., op. cit., p. 83-88.
} 
Tableau 1. Désignation de l'adversaire

\begin{tabular}{lcc}
\hline & Giscard d'Estaing & Mitterrand \\
\hline $\mathrm{Il}_{1}$ & 4,1 & 8,7 \\
Se son sa & 1,1 & 96 \\
Vous $1, \ldots$ & 72,1 & 491 \\
Votre, vos & 7,1 & 133 \\
Le nom & 10,4 & 15,2 \\
Autres & 5,2 & 4,1 \\
\hline Total & 100,0 & 100,0 \\
\hline
\end{tabular}

Fidèle à sa stratégie de 1974, dans plus de neuf cas sur dix, le président sortant dialogue avec son adversaire, le questionne, et quand il ne le vouvoie pas, il l'interpelle par son nom, procédé rhétorique classique qui fait monter la tension et ferme le cercle autour des jouteurs. Au contraire, M. Mitterrand semble vouloir sortir de cette situation aussi souvent que possible sans avoir l'air de rompre le combat. Alors que sept ans auparavant il avait plutôt répliqué sur le même registre à M. Giscard d'Estaing, en mai 1981, il parle de son adversaire sans s'adresser à lui dans plus de la moitié des cas. Les pronoms à la troisième personne, le nom, parfois même le vôtre lui servent de «coupe-circuit »pour s'adresser, par-dessus la tête de l'interlocuteur, aux auditeurs, c'est-à-dire aux véritables destinataires du discours. L'ensemble de ces procédés, un usage plus faible de vous 1 , tout concourt à donner aux propos du futur président un visage moins tendu ou, plutôt, suggère que la tension se situe non pas dans la relation je-vous 1 , mais dans celle que M. Mitterrand entretient avec son discours et, secondairement, avec les Français.

\section{Les Français}

Sans doute est-il banal de rappeler que les Français représentent les véritables destinataires du dialogue, mais cela semble pourtant contradictoire avec le poids très faible qu'ils ont dans le discours des deux candidats. Françaises et Français apparaissent une trentaine de fois chez M. Giscard d'Estaing et une quinzaine chez M. Mitterrand. En additionnant toutes les formes possibles sous lesquelles ils peuvent être désignés, tels quel les pronoms de la catégorie $i_{3} s_{3}$ ou vous $_{3}$, les dénominations particulières comme téléspectateurs, électeurs, concitoyens, peuple, etc., on obtient encore un total inférieur aux 
occurrences de la France (plus pays et nation), l'écart entre ces deux paradigmes étant maximum chez le futur président. Cette mise en scène par les hommes politiques de leur dialogue avec le pays (ou avec l'histoire) représente certainement l'une des principales figures de l'idéologie politique contemporaine. Mais si les Français sont des actants mineurs du discours, il n'en va pas de même de leurs votes ou plutôt de ce qui dans l'esprit des orateurs détermine ce vote, c'est-à-dire les intérêts économiques. On ne sera donc pas surpris par la grande importance qu'occupent, chez les deux adversaires, les mentions concernant les petites entreprises, artisans, agriculteurs, pêcheurs, employés, cadres, classes moyennes ainsi que les salaires, la fiscalité, les droits de succession... Le poids des intérêts « socioprofessionnels » apparaît considérable et pas seulement dans la seconde partie ${ }^{9}$.

Plus intéressante est la manière dont nos deux candidats essaient d'inclure les auditeurs dans leur propre énoncé pour que ceux-ci participent en quelque sorte à l'énonciation et s'identifient au locuteur. Deux procédés principaux sont utilisés ; les pronoms inclusifs et la forme didactique.

Nous appelons ici pronoms inclusifs le nous 3 et ses dérivés ainsi que les emplois du on qui équivalent à «tout le monde» (c'est-à-dire moi et les Français ${ }^{10}$ ). La figure 2 retrace l'usage qu'en font les deux adversaires.

\footnotetext{
${ }^{9}$ M. Charlot et I. Croizard en ont tenté un chiffrage pour la campagne de 1974, art. cité, p. 962-963.

${ }^{10}$ Pour une étude détaillée de ces procédés lors du débat de 1974, cf. Guilhaumou (Jacques), Lepage (Jeanine), «Etude du nous associatif dans un débat politique», Travaux de lexicométrie et de lexicologie politique, 2 , novembre 1977, p. 203-213.
} 
Figure 2. L'impersonnel et l'inclusion

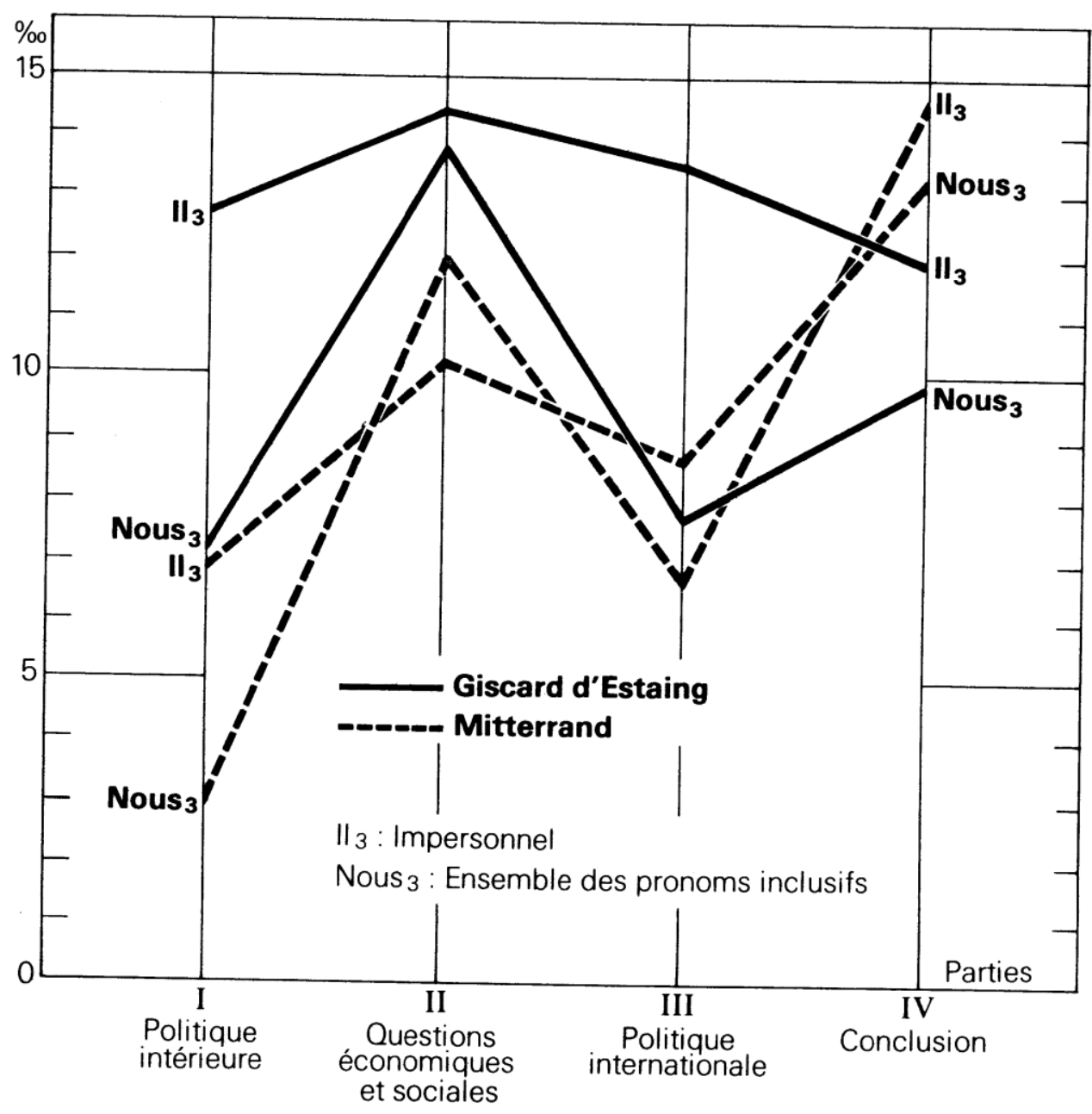

On remarque d'abord que leur fréquence est maximale en conclusion, ce qui souligne l'importance du procédé inclusif lorsque les orateurs se retrouvent face aux auditeurs. On constate également que l'emploi de ces pronoms évolue de façon parallèle chez les deux candidats et connaît une forte pointe dans la partie économique, ce qui confirme l'état d'esprit des debaters concernant le vote des Français. En dehors de l'exhortation qui ne se rencontre pas ici, l'inclusion répond à deux objectifs. D'une part, il s'agit d'associer l'auditeur à un verdict défavorable à l'adversaire. Ainsi, «aucune de vos réponses ne peut nous satisfaire» $(\mathrm{FM})$. D'autre part, l'inclusion peut être utilisée à propos d'un «constat» plutôt mélioratif pour le locuteur ou défavorable à son contradicteur: «nous n'avons jamais eu de croissance négative» (VGE). Le candidat socialiste est beaucoup plus sobre en procédés de ce genre. Il utilise plus volontiers le nous récapitulatif (nous 1 : moi et l'autre), ce qui 
s'explique puisque, comme nous le verrons, il se situe volontiers dans le discours en train de se tenir. D'autre part, chez lui, près de trois on sur quatre sont des équivalents de nous $_{2}$ (moi et d'autres: gouvernement, amis, etc.) ou de nous3 (moi et les Français), soit que M. Mitterrand adopte une tournure familière («figurez-vous qu'on l'a remarqué, nous aussi, le choc pétrolier») soit, plus souvent, pour donner un aspect impersonnel, donc «objectif», à l'opinion émise: «ou bien on adopte une politique dynamique de croissance, on essaie de reconquérir le marché intérieur...». Cette dernière forme est appelée didactique. M. Giscard d'Estaing l'utilise fréquemment. C'est pourquoi, dans ses interventions, quatre nous sur cinq sont inclusifs et, à l'opposé, dans une proportion à peine inférieure, les emplois du on suggèrent une disjonction plus ou moins péjorative (pas moi mais d'autres que je ne nomme pas...). Observons le fonctionnement de ce système dans le passage suivant :

«J'ai pris la décision ... d'approvisionner la France en uranium de telle manière que, quoi qu'il arrive ... nous avons notre production d'électricité nucléaire assurée pour cinq ans $^{11}$. Il peut y avoir une guerre, une crise ... Si nous continuons notre programme électronucléaire, nous avons l'électricité nationale la moins chère d'Europe. Nous aurons un avantage ... Ça vaut la peine, si on veut s'occuper du développement ... d'avoir le courant électrique le moins cher d'Europe. Donc je tiens à dire que c'est un intérêt national que de poursuivre ce programme. Si l'on arrête ..., c'est une décision négative, ce n'est pas une décision positive» (VGE).

L'ensemble du raisonnement se déroule entre $\operatorname{nous}_{3}$ (la France) et $i l_{3}$ ou le ça qui en est la dérivation (ça vaut la peine $=i l$ vaut la peine, etc.). Or qui «nous libère de la contrainte humiliante et inquiétante des producteurs de pétrole »? La décision de l'orateur qui est placée en tête du raisonnement, à la source de la situation favorable. Je ne réapparaît qu'au moment du verdict qui oppose on (l'adversaire innommé) à l'intérêt national. Du point de vue sémantique, l'apport du membre de phrase «je tiens à dire» est nul. Le lecteur peut faire l'expérience de le supprimer, il s'apercevra qu'apparemment rien ne change. L'irruption du locuteur a, cependant, une utilité. Elle permet de «cadrer» cette série d'énoncés à valeur universelle dans la polémique en cours, de placer je du côté positif et le vous, devenu on, du côté négatif. C'est elle qui assure le passage entre les deux formes rhétoriques fondamentales du discours politique. D'une part, la forme didactique qui fonctionne, comme nous l'avons dit, sans le je ou uniquement dans un emploi constatif peu fréquent (ici, «j'ai pris cette décision»); d'autre part, le discours polémique dont la dernière phrase citée représente un modèle. Elle juxtapose deux propositions dont la première se donne pour une transformation

\footnotetext{
${ }^{11}$ Membre de phrase répété deux fois selon un procédé très courant chez le président
} 
négative de propos prêtés à l'adversaire ${ }^{12}$. L'aspect polémique en est d'ailleurs renforcé par la transformation de vous en on. Dès lors, la relativement faible présence du je giscardien dans les deux premiers tiers de la soirée s'explique par la teinte très didactique des interventions de l'ancien président. Une preuve supplémentaire vient de l'utilisation importante du il impersonnel, autre grande forme du discours didactique (figure 2). Ce qui explique que $\mathrm{M}$. Mitterrand peut railler à plusieurs reprises le ton et l'allure professorale de son adversaire.

Mais, chacune des «leçons» de M. Giscard d'Estaing a pour but de contredire les positions de son interlocuteur et de se placer en quelque sorte du côté de la vérité. D'où la présence dans son discours de très nombreux verbes énonciatifs ${ }^{13}$ (50\% d'emplois en plus que chez M. Mitterrand) - comme je dis, j'indique, je note, j'observe... — introduisant généralement des opinions qu'on sous-entend partagées par l'auditeur ce qui, par là même, confirme cette forte volonté inclusive que nous notions plus haut. Au contraire, le candidat socialiste, s'il ne dédaigne aucun de ces procédés, y recourt beaucoup moins.

On peut mettre cette différence de tension dans la relation avec les locuteurs et les téléspectateurs, au compte du pressentiment qu'aurait eu le président sortant de l'opinion de la majorité des Français. En fait, la stratégie choisie par chacun des orateurs suffit à expliquer ce phénomène. En effet, M. Giscard d'Estaing a choisi, comme axe essentiel de ses interventions, la relation je-vous l, qui semble être dans la logique d'un duel télévisé mais comporte évidemment un risque : plus elle sera intense, plus elle tendra à exclure les tiers, spectateurs de l'affrontement. Elle ferme le cercle et il faut bien, d'une manière ou d'une autre, y réintroduire le véritable destinataire du message. C'est le rôle de la tension inclusive chez le président sortant. En revanche, M. Mitterrand a choisi de centrer son propos sur luimême et de ne pas se laisser enfermer dans la relation avec l'autre, qu'il objective autant que possible. Il peut donc se montrer plus économe en procédés inclusifs, éviter de sembler «faire la leçon» aux Français et s'épargner les nombreux changements de registre si frappants chez son adversaire. Il ne renonce pas pour autant à l'attaquer mais il a souvent recours, pour ce faire, à un procédé particulier qu'il est convenu d'appeler l'énoncé rapporté.

\footnotetext{
12 12. Dubois (Jean), «Avant-propos», in Marcellesi (Jean-Baptiste), Le Congrès de Tours, Paris, Le Pavillon, 1971, p. 16-17.

${ }^{13}$ Il s'agit de la catégorie des verbes qu'Austin appelle «expositifs», qui servent à expliquer une façon de voir, qui introduisent une argumentation ou une opinion. Ils impliquent une sorte de regard réflexif du sujet sur son énoncé, et sont généralement associés à une marque désignant le temps, le lieu ou la manière.
} 


\section{Le tiers exclu et l'énoncé rapporté}

La relation je-vous, qui domine ce débat, ne doit pas faire oublier qu'elle est d'abord le fruit du mécanisme simplificateur de l'élection présidentielle. En réalité, on sait que la situation des deux adversaires dépendait du report des voix qui s'étaient portées au premier tour sur les candidats éliminés. Or le débat a plutôt semblé fonctionner suivant le principe du tiers exclu : les différents $i l_{2}$ et $i l_{2}$ ont des occurrences très faibles et n'approchent un seuil significatif que dans la première partie. Il s'agit essentiellement de Jacques Chirac qui fut «présent» quelques instants, grâce au procédé classique de la citation dite «énoncé rapporté» en analyse de discours.

Il existe plusieurs types de citations. L'un d'eux consiste à inclure l'énoncé qu'on rapporte dans le discours sans disjonction de celui-ci. Par exemple, «j'indique que le lendemain du premier tour M. Chirac a indiqué qu'il voterait pour moi» (VGE). Ce procédé, qui a l'avantage de l'économie, présente toutefois une faiblesse ; l'auditeur peut toujours penser: «C'est vous qui le dites...». Pour pallier cet inconvénient, l'autre grand procédé consiste à rompre le discours pour laisser clairement place à une parole étrangère. Dans un texte écrit, cela se marque par un signe typographique tel que les guillemets. Pour la communication orale, on utilise des embrayeurs ${ }^{14}$ comme les pronoms: «vous dites: "Je dissoudrai tout de suite"» (VGE). Le danger demeure cependant que dans le flot du discours, l'embrayage passe inaperçu, ce qui arrive souvent à M. Giscard d'Estaing, comme en témoigne l'oubli par les sténographes des guillemets pour plusieurs de ses citations. Par exemple, à propos du projet socialiste que le président cite de mémoire sans lire le livre qu'il a devant lui. Au contraire, M. Mitterrand, moins sûr de lui ou, plus probablement, parce qu'il avait compris la nécessité de donner un support matériel à la parole rapportée, prenait souvent son papier et le lisait. Même quand ce n'était pas le cas, une pause dans le débit oratoire renforçait l'embrayage. De telle sorte que les sténographes ont gratifié pratiquement toutes ses citations (assez nombreuses) des guillemets d'usage attestant ainsi de l'effet produit sur un auditeur attentif. Pendant quelques instants, il a pu jouer des ambiguïtés chiraquiennes en évitant de laisser croire qu'il assumait personnellement le contenu de ce qu'il citait. Bien sûr, une exégèse un peu rigoureuse en montrerait la fragilité, et $\mathrm{M}$. Giscard d'Estaing s'y essaie parfois en évoquant la situation dans laquelle ces phrases ont été

\footnotetext{
14 Terme traduit de l'anglais shifter qu'utilisé Jakobson pour désigner la classe des unités grammaticales dont le sens varie avec la situation et le contexte dans lequel ils sont employés: mode, temps, personne, etc. (Cf. Jakobson (Roman), «Les embrayeurs, les catégories verbales et le verbe russe», Essais de linguistique générale, Paris, Le Seuil, 1970, p. 176-1\%)
} 
prononcées. Mais cette attitude, fondée en histoire, par exemple, est bien faible dans un débat politique. Alors, à d'autres moments, il menace («j'en ai une sous les yeux, je ne la lirai même pas tellement elle est sévère») ou admoneste : «ne recherchons pas les citations du passé dans lesquelles vous vous complaisez»; mais à aucun moment il ne trouve la parade. Or l'ensemble des énoncés de M. Chirac ou de M. Giscard d'Estaing lui-même, qui furent ainsi rapportés par M. Mitterrand, ont occupé une part importante du temps que celuici a consacré au procès de son adversaire, que ce soit à propos des querelles de l'ancienne majorité, du bilan économique et social du septennat ou même de la rencontre de Varsovie avec M. Brejnev. Il est probable que M. Mitterrand a voulu multiplier les citations chiraquiennes ou les références au général de Gaulle pour gagner des voix gaullistes, mais il aura semblé ainsi laisser à d'autres le soin de juger le passé, pour se consacrer à la défense de son programme et pour se tourner vers l'avenir. De plus, l'intervention simulée d'un tiers dans le débat faisait d'autant baisser la tension je-vous et brisait le cercle dont nous parlions plus haut.

L'ensemble du jeu des actants principaux fait donc apparaître deux stratégies discursives bien typées et fortement divergentes. Cette situation nouvelle par rapport à 1974 va rejaillir sur l'ensemble du débat.

\section{RÉCIT ET DISCOURS}

L'autre grande famille d'indices, par lesquels se matérialise l'énonciation en œuvre dans un discours, concerne les verbes à la fois sous l'angle de leur aspect, de leur temps, des «marques» qui les accompagnent, comme les adverbes ou prépositions de temps, lieu, manière..., ainsi que dans l'usage des auxiliaires modaux tels que pouvoir, devoir, etc. L'étude de ces indices va nous permettre de compléter le tableau des stratégies poursuivies par les deux candidats.

\section{L'aspect des verbes}

Suivant leur construction ou leur nature, les verbes peuvent être classés en deux catégories. D'une part, l'accompli regroupe les formes verbales avec être ou avoir ou commutables avec eux : «Vous n'êtes pas le président de la République ici»(FM). Ces 
verbes marquent «un état, un accompli, une distance, une absence de tension» ${ }^{15}$. Au contraire, le non-accompli est construit ou peut se construire avec des auxiliaires comme faire, pouvoir, vouloir, savoir, devoir, falloir... Ils indiquent une tension plus ou moins grande du sujet face à l'interlocuteur ou à son propre discours ainsi qu'une absence de distance, un énoncé fortement assumé: «Je dissoudrai quand je le déciderai» (FM), «si vous agissez comme vous le dites»(VGE). Nous avons donc recherché le poids relatif de ces deux catégories en ne retenant que les verbes ayant comme sujet le locuteur et son adversaire, seuls ou inclus avec d'autres par le jeu des pronoms.

Au total, le rapport accompli - non-accompli dépasse un pour trois, ce qui marque le caractère fortement tendu de ce type d'échanges. A l'intérieur de ce rapport d'ensemble, les deux contradicteurs présentent une image presque inversée : M. Giscard d'Estaing marquant une préférence relative pour les accomplis, et M. Mitterrand pour les non-accomplis. Ce qui confirme la distance plus grande que manifeste le président sortant envers son discours nettement didactique. Cependant, l'image se brouille quand on répartit les verbes suivant qu'ils ont pour sujet le locuteur ou son adversaire.

Chez M. Giscard d'Estaing, le je est plutôt accompli et le vous inaccompli, répartition qui s'inverse chez M. Mitterrand (en tenant compte du rapport établi ci-dessus et qui nous sert de norme de référence). Le président sortant, lorsqu'il se pose en sujet de son discours, tend à gommer la tension et la renforce plutôt quand il parle de l'autre. Souvent, la tension qui émane de ses propos est assumée grâce à un procédé inclusif: «il faut que nous poursuivions notre effort $»$; de telle sorte que le locuteur paraît prendre une distance assez grande envers le monde. En revanche, les énoncés portant sur le vous sont fortement inaccomplis, ce qui reporte la tension vers la relation que l'autre entretient avec les actants du discours, qui sont, en dehors du je, le Parlement, les électeurs, la Constitution, les partis, les entreprises, etc. Or M. Mitterrand semble calquer son énonciation sur un modèle assez semblable. Pour le vous, il utilise plutôt des accomplis, ce qui traduit bien la volonté d'objectiver l'autre, et utilise avec je un net excédent, par rapport à notre moyenne théorique, de verbes d'action et surtout d'auxiliaires modaux. Le schéma général qui se dégage ainsi apparaît curieusement semblable. Les deux adversaires semblent d'accord pour placer M. Giscard d'Estaing du côté de la distance, de l'être (ou de l'avoir) et pour situer M. Mitterrand plutôt vers l'inaccompli, la tension, le faire.

\footnotetext{
${ }^{15}$ Dubois (Jean), art. cité, p. 107.
} 
Bien sûr, on aura deviné que cet accord pour le moins curieux correspond en réalité à deux messages opposés que va mettre en évidence le rôle du temps dans les deux stratégies discursives.

\section{Le rôle du temps}

Le présent grammatical semble être le temps de prédilection de M. Mitterrand : près de $62 \%$ de ses verbes contre la moitié chez son adversaire. M. Giscard d'Estaing utilise beaucoup plus le passé $(30 \%)$ que le candidat socialiste $(10 \%)$, et ce dernier préfère nettement le futur (28\% contre 19,5\%). Cette distribution ne manque pas d'être suggestive ; on peut aisément y voir M. Mitterrand assumant mieux son discours et envisageant facilement le futur. Au contraire, il y a chez le président sortant des déplacements plus fréquents dans le temps mais surtout vers le passé...

Cependant, une telle vision des choses ignorerait la complexité des phénomènes d'énonciation. En effet, ce qu'on appelle le temps grammatical n'entretient pas, dans le langage, de rapport simple et direct avec le temps physique, la temporalité existentielle. En réalité, dans ce débat, il y avait nettement trois temps superposés :

- D'une part, le temps intérieur au discours qui permet d'enchaîner ce qui a été dit avec ce qui va suivre. Ainsi, «j'ai dit tout à l'heure» ou «nous y reviendrons» qu'emploient fréquemment les deux interlocuteurs. Mais, en réalité, quel que soit le temps grammatical, on s'aperçoit qu'il s'agit toujours d'«ici et maintenant» suivant l'expression d'Emile Benveniste $^{16}$. La preuve en est que les locuteurs emploient indifféremment le présent, le passé et même le futur (par exemple, «vous dites...» au lieu de «vous avez dit») pour parler des prises de position, des programmes. C'est pourquoi la lecture du débat montre que cet « ici et maintenant » se dilate au-delà de la soirée du 5 mai 1981. Il s'étend à l'ensemble de la campagne et situe l'affrontement électoral dans l'ordre du discours ${ }^{17}$. Les temps grammaticaux, les dates, les adverbes jouent alors une fonction assez anaphorique ${ }^{18}$.

- D'autre part, le temps chronique qui ne fonctionne réellement que pour l'avant et l'après-campagne. Le dix mai représente dans l'esprit des deux candidats la barre où l'on

\footnotetext{
${ }^{16}$ Benveniste (Emile), «Les relations de temps dans le verbe français», op. cit. p. 237-250.

${ }^{17}$ Ibid.

${ }^{18}$ En linguistique, on appelle anaphore la relation interne au discours qui s'établit entre deux parties de celui-ci (par exemple les pronoms qui renvoient à quelque chose ou à quelqu'un de déjà nommé). La déixis (avec son dérivé : déictique) est l'acte de renvoyer à une situation extérieure au discours grâce à un embrayeur ou à une marque telle que date, référence de lieu, etc.
} 
bascule du présent au futur. Pour parler de l'après-dix mai, le temps grammatical normal sera le futur (en dehors de la concordance des temps), mais le présent est parfois utilisé grâce à des marques déictiques — dates ou formules telles que «si vous êtes élu » — qui indiquent la place future de l'événement décrit. Il en est généralement de même pour l'avantcampagne. Grâce à ces embrayages, nous sortons du discours proprement dit pour entrer dans plusieurs systèmes qu'on nommera récit, fiction, théorie, histoire, etc., suivant que la communication sera orale ou écrite et selon le genre adopté ${ }^{19}$.

- Enfin, il existe une dernière catégorie qui vise à signifier la permanence, l'intemporalité. Ainsi, lorsque M. Mitterrand s'exclame : «je suis quelqu'un qui a toujours respecté la loi» ou encore quand M. Giscard d'Estaing lui rétorque «vous gérez le ministère de la parole», il s'agit de suggérer un caractère intrinsèque à la personnalité qui échappe au temps physique. Les hommes politiques sont friands de ce procédé et nos deux candidats n'échappent pas à la règle.

Dès lors, on comprendra que, par rapport au temps grammatical, les marques déictiques représentent un bien meilleur indice de la relation qu'entretient le locuteur avec le temps et avec le monde qui l'entoure. L'intérêt de cette recherche se devine aisément : plus le discours étudié comportera de telles marques, plus il sera facile de le discuter, de l'infirmer, de le relativiser. Au contraire, le discours idéologique tend toujours à masquer les conditions de sa production, qui viendraient contredire sa prétention universalisante ${ }^{20}$.

Le démonstratif constitue le modèle de la marque et le meilleur exemple de ses ambiguïtés. En effet, seuls certains emplois du démonstratif sont déictiques («c'était hier», donc le 4 mai 1981), alors que d'autres servent à établir des liaisons internes au discours, c'est-à-dire des anaphores («ce qui veut dire»). De plus, un usage répété de l'une de ces formes peut avoir plusieurs significations. Par exemple, M. Giscard d'Estaing utilise plus le $c ̧ a$ anaphorique que son concurrent : est-ce à dire que son discours soit plus replié sur luimême, contrairement à tout ce que nous avons montré jusqu'ici ? En réalité, cette particularité s'explique par le style de l'ancien président qui, parlant rapidement à l'aide de propositions peu complexes mais très imbriquées, se heurte à des problèmes de répétition et de coordination, qu'il résout, grâce à des formules comme «c'est-à-dire» ou «cela signifie», etc. Il pourrait les éviter en construisant ses phrases de façon moins heurtée et plus

\footnotetext{
${ }^{19} \mathrm{Cf}$. sur ce point une «typologie des discours» proposée par Simonin-Grumbach (Jenny) in Kristeva (Julia) et al., Langue, discours, société, Paris, Le Seuil, 1975, p. 85-121. La distinction se révèle assez complexe. Ce n'est pas le lieu de reprendre cette discussion. Cependant, il faut se souvenir que les catégories employées ici n'ont pas de valeur certaine.

${ }^{20}$ Reboul (Olivier), Langage et idéologie, Paris, PUF, 1980.
} 
monotone, comme le fait son adversaire, mais il lui faudrait alors reporter sur la diction cette perte d'emphase au niveau du texte. L'étude du style des candidats n'est pas dans notre propos, nous voulions simplement montrer ici la complexité de ces phénomènes, qui interdit d'utiliser des indicateurs uniques sortis de leur contexte. Il faut recenser l'ensemble des marques de temps ou de lieu quelles qu'en soient les formes (adverbes, prépositions, dates...).

Nous nous sommes intéressé aux marques de temps qui sont les plus nombreuses (un décompte partiel nous a montré que les marques de lieu obéissent aux mêmes tendances). Globalement, les deux protagonistes paraissent à égalité : un peu plus d'un tiers de leurs phrases contiennent des marques temporelles. En écartant celles qui concernent la campagne électorale - dates des déclarations, des scrutins... dont nous avons dit la fonction anaphorique -, on constate que moins du quart des phrases sont situées dans le temps, hors de ce vaste discours, avec un net avantage à M. Giscard d'Estaing, M. Mitterrand se concentrant plus sur le présent discursif. Il semble que nous retrouvions, dans cette faiblesse de l'ancrage temporel, un autre aspect du masquage évoqué ci-dessus. Mais comme il est relativement commun aux deux locuteurs, il faut sans doute l'attribuer au discours politique en général ou, plus vraisemblablement, aux conditions particulières d'un tel débat.

Cependant, si masquage il $\mathrm{y}$ a, il se révèle inégalement réparti selon les problèmes traités. En admettant que l'ancrage dans le temps varie en fonction inverse de la déixis, on peut tracer pour les deux candidats une courbe de leur comportement au cours du débat. Cette courbe est décroissante chez M. Mitterrand et croissante pour son adversaire. Autrement dit, M. Giscard d'Estaing avait beaucoup plus à cacher en matière de politique intérieure ou d'économie qu'en politique extérieure. En revanche, le candidat socialiste se trouve dans une situation inverse mais encore plus marquée puisqu'il remporte de très loin à la fois la palme du discours le mieux ancré dans le temps et dans l'espace lorsqu'il traite de politique intérieure ainsi que, avec sa conclusion, celle du texte le plus intemporel de la soirée. Le saut est particulièrement brusque puisqu'en passant de la politique à l'économie, le discours de M. Mitterrand perd plus de $60 \%$ de ses marques temporelles, ce qui contribue à donner aux positions économiques qu'il défend un caractère théorique que n'a pas manqué de relever son contradicteur.

A l'intérieur de ce phénomène d'ensemble, nous pouvons reprendre notre distinction ternaire (avant-maintenant-après) et distinguer suivant que la marque s'applique au locuteur ou à l'adversaire. Pour chacun des candidats, un schéma temporel se dégage, que nous résumons dans la figure 3. 
Figure 3. Orientation temporelle du locuteur et de son adversaire dans leur discours (d'après les marques déictiques en \%).
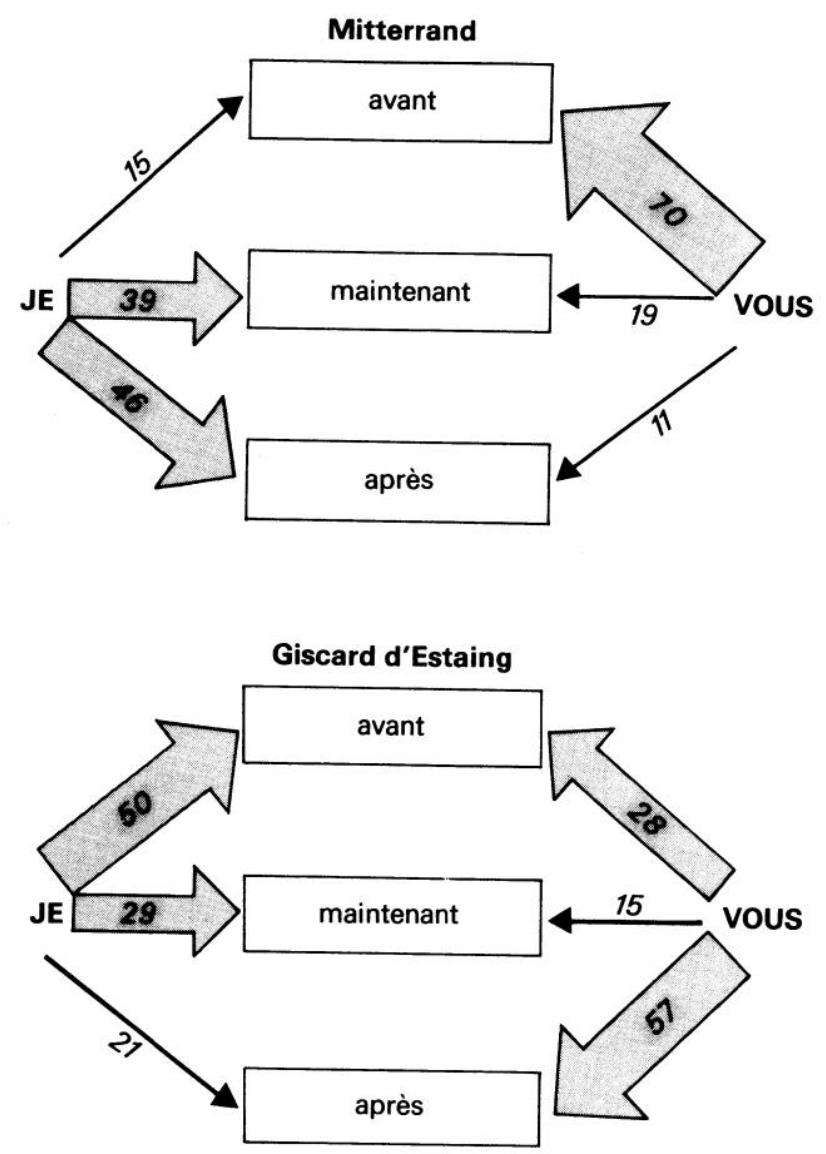

Cette figure permet maintenant de chiffrer avec certitude les principales tendances que nous avons pressenties auparavant :

- M. Giscard d'Estaing est massivement situé dans le passé aussi bien par son adversaire que par lui-même (la moitié des marques le concernant dans son propre discours). Ce qui n'est pas surprenant si l'on songe que le président sortant plaide pour la continuité. Il se doit donc de paraître assumer sa gestion passée : «Je prends la responsabilité de ce qui a été fait», «Je ne fuis aucune responsabilité», dit-il à plusieurs reprises. Nous avons vu que la réalité de son discours est bien différente puisqu'il s'efface les deux tiers du temps et parle surtout de l'autre. C'est la raison pour laquelle, si les deux candidats situent plutôt M. Mitterrand dans le futur, le président sortant le fait avec plus d'insistance encore que l'intéressé lui-même (57\% contre $46 \%$ ). 
- M. Mitterrand reste beaucoup plus dans le vaste discours de la campagne électorale (maintenant) que ce soit pour le je ou pour le vous. De ce fait, la grande majorité des marques sortant de ce «présent de l'énonciation» se trouvent dans la bouche de son adversaire.

Pour reprendre la distinction discours-récit, nous pouvons dire que le candidat socialiste se situe plus près du discours et son contradicteur nettement vers le récit. On doit cependant se garder d'en déduire que les propos de M. Mitterrand sont forcément plus idéologiques que ceux de M. Giscard d'Estaing car l'organisation de la temporalité est plus simple chez le candidat socialiste (l'autre avant, moi maintenant et après), alors que l'ancien président, au milieu d'un discours fortement polémique, enchevêtre plusieurs récits portant sur l'autre ou sur lui-même avant comme après (l'un d'entre eux domine toutefois, celui portant sur vous après). Il lui faut donc utiliser des formules comme «si vous êtes élu», afin de suggérer qu'on est dans la fiction, et employer un plus grand nombre de marques, hors du maintenant, pour «embrayer» d'un récit à l'autre ou simplement pour rappeler à son auditoire le contexte de son énoncé. On devine la complexité d'un tel exercice intellectuel ainsi que les risques qu'il comporte et que nous avons déjà évoqués à propos des énoncés rapportés.

Ce canevas ne représente toutefois qu'une moyenne générale. Pour rendre compte des mouvements internes, nous avons comparé la situation relative dans le temps physique du je et de l'autre dans chacun des discours à l'aide d'un indicateur simple ${ }^{21}$ :

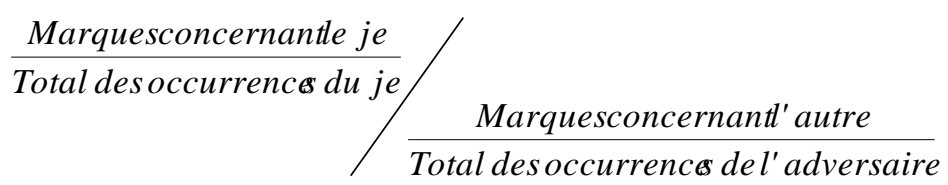

Si la valeur obtenue dans un passage donné dépasse l'unité, cela indique qu'à cet endroit le locuteur tend à mieux se situer dans le temps ainsi qu'à en abstraire son adversaire, et inversement, lorsque l'indicateur est inférieur à 1 . Sur l'ensemble du débat, le résultat est de 1,70 pour M. Giscard d'Estaing et de 0,60 pour M. Mitterrand (tableau 2) : le président sortant bénéficie d'un bien meilleur ancrage temporel dans son propre discours comme dans celui du candidat socialiste. En quelque sorte, dans les interventions de M. Giscard d'Estaing, un moi fortement situé dans le temps fait le procès d'un vous plus discursif, ce que le président sortant résume dans des formules comme «vous gérez le ministère de la parole

\footnotetext{
${ }^{21}$ Pour ces décomptes, cf. plus haut (encadré et figure 1).
} 
... moi je gérais la France »; modèle qui se trouve à la source du système temporel en œuvre dans ses propos. En revanche, dans le discours de M. Mitterrand, c'est un moi assez intemporel qui s'oppose à un vous mieux situé (nous savons maintenant qu'il s'agit du passé). Ce résultat global, s'il fournit une clef essentielle de compréhension du débat, cache cependant des variations importantes suivant les problèmes abordés (tableau 2).

Tableau 2. Ancrage relatif dans le temps du je et du vous

\begin{tabular}{lcc}
\hline Parties & Giscard d'Estaing & Mitterrand \\
\hline I. Politique intérieure & 0,7 & 3,3 \\
II. Questions économiques et sociales & 2,7 & 0,4 \\
III. Politique internationale & 2,4 & 2,7 \\
IV. Conclusion & 1,2 & 0,6 \\
\hline Moyenne & 1,7 & 0,7 \\
\hline
\end{tabular}

- Lorsqu'il s'agit de la politique intérieure française, les deux adversaires situent remarquablement mieux M. Mitterrand que M. Giscard d'Estaing. Nous sommes ici dans une situation inverse par rapport à la loi générale qui vient d'être dégagée. Pourquoi ce renversement ? D'une part, le candidat sortant, dans les brefs passages qu'il consacre à la situation de sa majorité, tente de dédramatiser, non seulement en s'effaçant, comme nous l'avons montré plus haut, mais aussi en temporalisant assez peu son propos. Du même mouvement, il centre ses interventions sur l'adversaire et tente de faire apparaître des contradictions dans ses positions politiques ; d'où les nombreuses marques concernant la dissolution et les législatives. D'autre part, M. Mitterrand semble prendre la balle au bond et se situer fortement dans cet après. Au contraire, il parle peu de son adversaire, souvent de manière indirecte et faiblement temporalisée («vous n'avez pas la majorité»). La figure 3 suggère que le résultat de ce double mouvement est de faire disparaître $M$. Giscard d'Estaing de la scène politique future qui semble entièrement occupée par le candidat socialiste. L'ancien président ne recherchait certainement pas cette impression mais il la provoque par son choix discursif.

- La relation s'inverse dans la partie économique. Le procès que M. Giscard d'Estaing intente à son adversaire domine encore mais appartient ici à l'ordre du discours puisqu'il est en moyenne très peu temporalisé contrairement à la défense de sa propre action et, en mineure, de ses projets qu'il mène plutôt sur le mode du récit. Or les arguments du candidat 
socialiste sont encore plus détachés du temps physique, les seules marques utilisées pouvant toutes se résumer à «si je suis élu» et «pendant mon mandat». Ses positions économiques, bien qu'encore fortement assumées, sont beaucoup moins situées que ses projets politiques. La baisse du je dans la seconde partie (figure 1) confirme cette distanciation relative de $\mathrm{M}$. Mitterrand par rapport à l'économie. En revanche, quand il critique le septennat précédent, il utilise volontiers des chiffres, des dates et des citations. Là encore, il semble donc y avoir un consensus - inverse du précédent - pour placer M. Giscard d'Estaing plutôt dans le récit et M. Mitterrand du côté du discours. Naturellement, chacun des candidats affecte des valeurs inverses au général peu temporalisé (le programme du futur président) et au singulier situé (le sortant, son action et ses projets concernant le chômage que M. Mitterrand ne daigne d'ailleurs pas discuter). Bien que non voulu par M. Giscard d'Estaing, l'effet paraît le même que précédemment.

- Pour la partie concernant la politique internationale, nous avons affaire à un schéma plus simple puisque les valeurs obtenues dans la figure 3 sont sensiblement les mêmes pour les deux adversaires: je est environ deux fois et demie mieux situé que l'autre, M. Mitterrand accusant un peu ce trait. Chez M. Giscard d'Estaing, pour la première fois, le je passé s'affirme alors que le vous/ après disparaît. Autrement dit, l'ancien président n'assume véritablement que sa politique étrangère. On retrouve là son jugement implicite sur les forces et les faiblesses de son septennat. Il est donc possible de supposer que sa volonté, affirmée d'entrée de jeu et réaffirmée à plusieurs reprises, de consacrer le débat à l'avenir représente aussi un moyen commode pour ne pas s'étendre trop sur le bilan économique et social puisque cette volonté le quitte lorsque l'on passe aux questions internationales... Quant à M. Mitterrand, un mouvement parallèle le conduit, lui aussi, à privilégier le je avant et à délaisser quelque peu le discours au profit du récit et de l'intemporel. Pourquoi ce retournement chez les deux candidats? Une analyse thématique de cette partie montre que, sauf dans une certaine mesure à propos de la République Centrafricaine, il s'est agi en fait de politique intérieure : le vote des éleveurs, des agriculteurs, des producteurs méditerranéens, des pêcheurs «venus de chez nous», des juifs, des Français de l'étranger «qu'il faut protéger», des militaires «exposés dans leur vie» à cause de la Lybie... et bien sûr du vote gaulliste (la défense). Le locuteur date, localise ses propres actes et déclarations en faveur de ces catégories, c'est-à-dire qu'il présente un récit circonstancié de son action «au service de la France». Inversement, il attaque son adversaire de manière abstraite et multiplie les petites phrases : «M. Mitterrand n'a pas voté un centime pour la défense de la France», «on ne doit pas agir comme un prophète» (VGE), «Vous avez manqué de fermeté, cela vous arrive assez 
souvent» (FM), etc. Nous sommes donc dans un discours électoral classique avec, bien en évidence, la conception que les candidats se font du vote des Français. Qu'ils agissent ainsi de façon délibérée, nous en trouverons confirmation dans la conclusion de M. Mitterrand, texte évidemment longuement préparé, où il avoue qu'il aurait «aimé parler davantage du Tiers Monde, de l'Amérique centrale ... du rôle de la France» et, plus loin, du «droit des peuples à disposer d'eux-mêmes»... ${ }^{22}$.

- Pour les conclusions, le tableau 2 donne des valeurs proches des moyennes générales. Comme les textes ne sont pas improvisés, ce résultat prouve que notre indicateur révèle assez fidèlement la stratégie choisie par chacun des protagonistes. Par exemple, $\mathrm{M}$. Giscard d'Estaing, pour conclure, résume son schéma décrivant l'alternative économique et sociale qui s'offrirait aux Français le 10 mai, mais l'oublie à propos des Affaires étrangères ! Le $j e$, enfin, ne se masque plus et se situe mieux dans le temps par rapport à son adversaire («il n'a pas changé ... moi, pendant ces sept ans, je me suis transformé»). M. Mitterrand, lui aussi, laisse apparaître sa stratégie qui consiste à opposer un court récit de l'échec de son adversaire - avec des phrases brèves, une image choc (la voiture dans le fossé) - et un long discours d'où le je, la temporalité disparaissent quasiment au profit du il impersonnel, de la liberté $^{23}$ et, secondairement, de la France et de l'histoire, pôles essentiels de la symbolique du futur président.

Deux comportements discursifs bien typés se dessinent maintenant. Reste à comprendre ce qui motive cette différence dans l'énonciation. C'est ce que nous apprendra l'étude des modalités discursives.

\footnotetext{
${ }^{22}$ Rappelons qu'à la fin de cette discussion, M. Mitterrand a cédé deux minutes à son adversaire pour parler du Moyen-Orient.

${ }^{23}$ Le mot «liberté» se trouve au cœur du discours socialiste. C'est lui qui déclenche le plus grand nombre d'associations avec d'autres mots ; cf. Gerstlé (Jacques), Le langage des socialistes, Paris, Stanké, 1978, p. 3841.
} 


\section{Vouloir faire ou savoir faire?}

La « modalisation ${ }^{24}$ de l'énoncé représente le dernier grand type d'indices qui permettent de reconnaître l'énonciation de la subjectivité dans le discours. Au cœur de ce phénomène se trouvent incontestablement les auxiliaires modaux (vouloir, devoir, pouvoir, savoir et falloir), car ils sont associés à un verbe factitif ou existentiel et suggèrent ainsi le genre de relations qu'entretient le locuteur avec son vis-à-vis ou avec son objet (volonté, obligation, permission, désir, connaissance, etc.). C'est pourquoi l'étude de leurs emplois au cours du débat se révèle particulièrement éclairante.

Nous avons relevé les contextes de ces cinq verbes et de leurs multiples synonymes (ainsi, pour vouloir: j'entends, je compte, j'ai envie...; pour devoir : avoir le droit, interdire, permettre de, etc.). Ces formes très diverses apparaissent près de 500 fois au cours du débat, ce qui dépasse largement l'utilisation qu'en font, par exemple, les résolutions des congrès communistes ou socialistes. Faute de points de repère, il nous est cependant difficile de dire si ce discours tendu appartient en propre aux deux locuteurs ou si, comme c'est probable, la forme même du débat le leur impose. Là encore, ce seront les différences entre MM. Giscard d'Estaing et Mitterrand qui nous serviront de guides.

En valeur absolue, M. Mitterrand utilise une vingtaine de modalités de plus que son adversaire mais, comme il a moins parlé, la différence réelle est plus grande: on trouve, en moyenne, plus d'une modalité pour deux phrases chez le candidat socialiste (55\% contre $40 \%$ chez M. Giscard d'Estaing ${ }^{25}$ ). Enfin, le décompte fait apparaître que, sur l'ensemble du débat, ce dernier modalise surtout le vous, et M. Mitterrand le je. Nous retrouvons ici l'asymétrie plusieurs fois relevée au cours de cette analyse : le candidat socialiste assume la tension qui se dégage de son discours, alors que l'ancien président tente de la reporter sur l'autre. Le passage dans lequel M. Giscard d'Estaing envisage son avenir politique nous fournit une bonne illustration de ce déplacement:

\footnotetext{
${ }^{24}$ Cette notion qui provient de l'étude de l'intonation est assez polysémique. A la limite, elle en vient à désigner tout choix libre du sujet qui, sans atteindre le contenu sémantique de l'énoncé, permet à l'énonciation des variations importantes (jeu des adverbes, types de phrases, utilisation des modes verbaux, transformations passives facultatives, etc.). L'ensemble donne, en quelque sorte, la «longueur d'onde» sur laquelle est émis le message (Jean Dubois). Une acception plus restreinte, que nous retenons ici, limite ce terme à l'utilisation des cinq auxiliaires modaux. Cf. «Modalités, logique, linguistique, sémiotique». Langages, 43, septembre 1976.

${ }^{25}$ Il s'agit d'un calcul théorique. En réalité, certaines phrases combinent plusieurs modalités. Le nombre des phrases a été choisi comme dénominateur commun pour des raisons méthodologiques. En prenant le nombre de mots prononcés comme base de calcul, les différences sont un peu moins nettes mais tout aussi significatives (la phrase de M, Mitterrand est en moyenne plus longue).
} 
«Je note que la totalité des dirigeants de la majorité, des parlementaires, avaient indiqué qu'ils voteraient désormais pour le président de la République. Donc la question de ma majorité, et quand je dis "ma" majorité ce n'est pas un "ma" possessif, est réglée. Il n'y aura pas de dissolution. Un gouvernement sera constitué. Il comportera des personnalités représentatives ... et il se mettra au travail pour adopter les premiers textes concernant l'emploi ... que je voudrais voir figurer à la session parlementaire de printemps».

On y voit le je s'effacer grâce à des substitutions (président de la République à la place de moi), à des transformations passives avec suppression du complément d'agent (dans le passage concernant le gouvernement) ou grâce à l'usage du il impersonnel («il n'y aura pas de dissolution» pour «je ne dissoudrai pas»). Naturellement, le lecteur aura remarqué le scrupule hautement significatif qui saisit l'orateur lorsqu'il se rend compte qu'il a usé du possessif à propos de la majorité d'alors. Dans ce passage, la tension disparaît également du fait de l'utilisation de verbes accomplis là où il aurait fallu des inaccomplis («les parlementaires veulent que j'ai la majorité» devient «la question est réglée»). Même phénomène à propos du gouvernement dont la composition se présente comme un fait brut sans formation ni formateur. «Je note» se donne donc pour un constat, alors qu'il introduit une manière très personnelle de voir les choses : on aura reconnu le phénomène inclusif dont nous avons dit l'importance chez l'ex-président. Ainsi, la lutte contre le chômage demeure la seule chose assumée par le locuteur ; le reste semble appartenir à un ordre naturel, «allant de soi». En revanche, le passage qui suit, beaucoup plus long, concerne la critique des projets de M. Mitterrand :

«Nous ne sommes pas dans un régime présidentiel ou personnel. Vous m'avez fait des reproches à cet égard ..., vous en avez fait encore plus au général de Gaulle ... La Constitution dit ... Si vous agissez ainsi que vous le dites ... votre gouvernement ne pourra rien faire, il n'aura le droit de ne rien faire, il devra attendre ... Nous n'avons pas en France un système tel qu'un président, vous ou moi ou le général de Gaulle dans le passé, puisse nommer ... Il ne pourra faire qu'une chose, c'est ce qu'on appelle — vous connaissez la formule - expédier les affaires courantes, etc. ».

Ici l'énoncé, très didactique, semble pris en charge par le nous inclusif (les Français, la France) mais aussi par le général de Gaulle, cité deux fois, par la Constitution et même par M. Mitterrand. L'ensemble des modalités et le ne pas faire sont mobilisés sur vous 1 et votre gouvernement pour suggérer non seulement la tension mais l'impossibilité. On a donc une forte opposition entre l'être, l'accompli (Giscard élu) et l'inaccompli, l'impossible (Mitterrand élu), dans une forme très achevée qui utilise toutes les ressources de 
l'énonciation. Au fond, le schéma de base est donné par la célèbre formule « moi ou le chaos», elle-même dérivée de ordre/désordre, qui, faute de pouvoir être exprimée ouvertement, doit se suggérer dans le moment même où l'on prétend analyser la situation objectivement et sans passion : procédé systématique chez l'ancien président. Au contraire, si M. Mitterrand ne répugne pas à s'en servir - par exemple à propos de la division des partis de gauche ou des nationalisations, ce qui n'est pas sans signification - cela fait figure d'exception. En règle générale, le je assume et modalise fortement son énoncé : l'intervention du candidat socialiste qui précède le passage cité représente, de ce point de vue, un modèle auquel nous renvoyons le lecteur.

A une différence dans la nature de la tension correspond donc une divergence très nette dans l'usage de la modalisation. Nous l'avons synthétisée grâce à deux figures (4 et 5) où, pour chacun des orateurs, le poids relatif de la modalité considérée (rapport du nombre d'emplois et du nombre de phrases) sert de définition au polygone référentiel de l'autre. Comme on le voit, la première caractéristique réside dans le goût de M. Giscard d'Estaing pour la transformation négative de devoir, pouvoir, savoir et falloir ainsi que dans la répugnance égale que lui manifeste son concurrent. D'autre part, le calcul confirme ce que montrent les graphiques : vouloir, pouvoir et ne pas pouvoir sont, dans cet ordre, les modalités les plus spécifiques au candidat socialiste ; les transformations négatives de savoir, falloir et devoir représentent celles du président sortant. 
Figure 4. Modélisation du discours de M. Giscard d'Estaing par rapport à celui de M. Mitterrand.

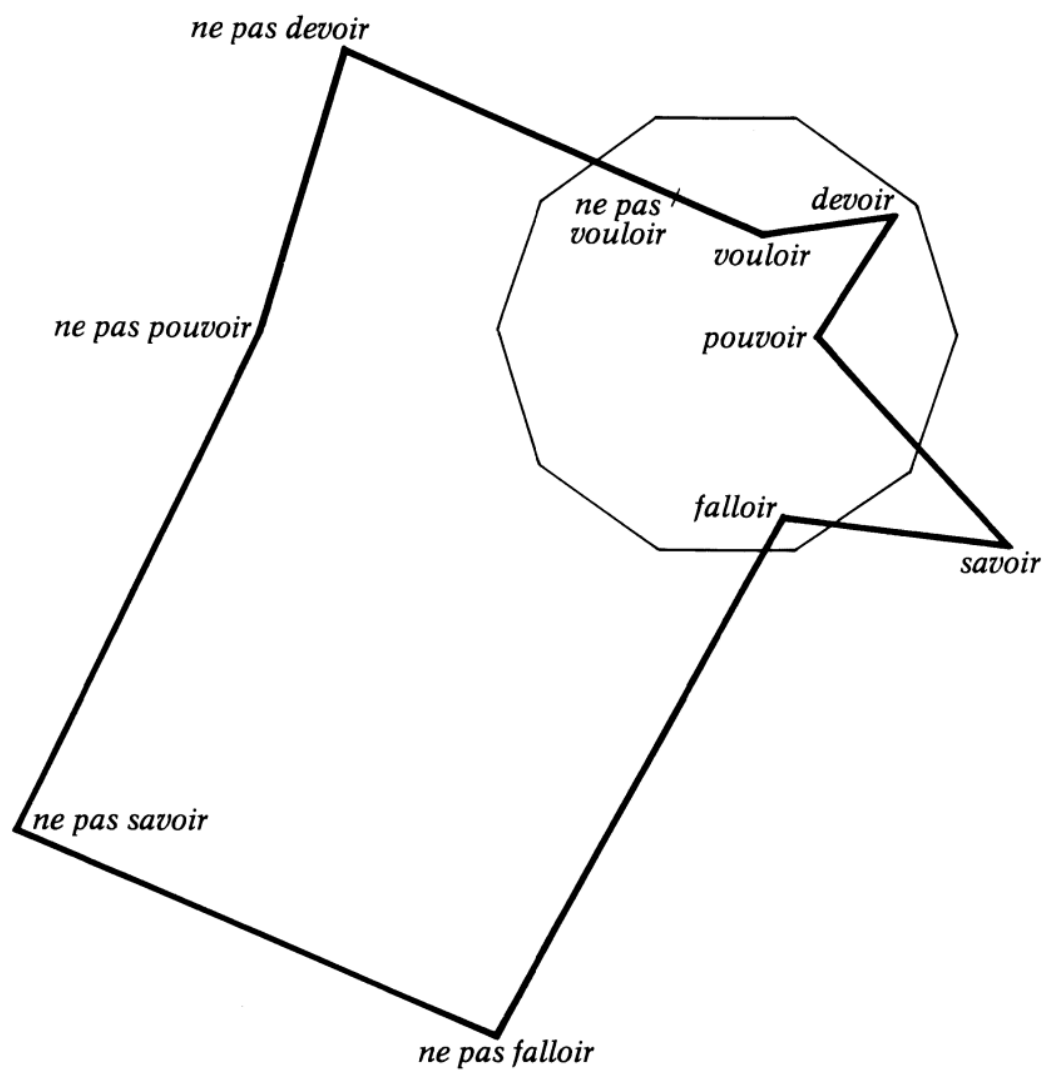

Figure 5. Modalisation du discours de M. Mitterrand par rapport à celui de M. Giscard d'Estaing.

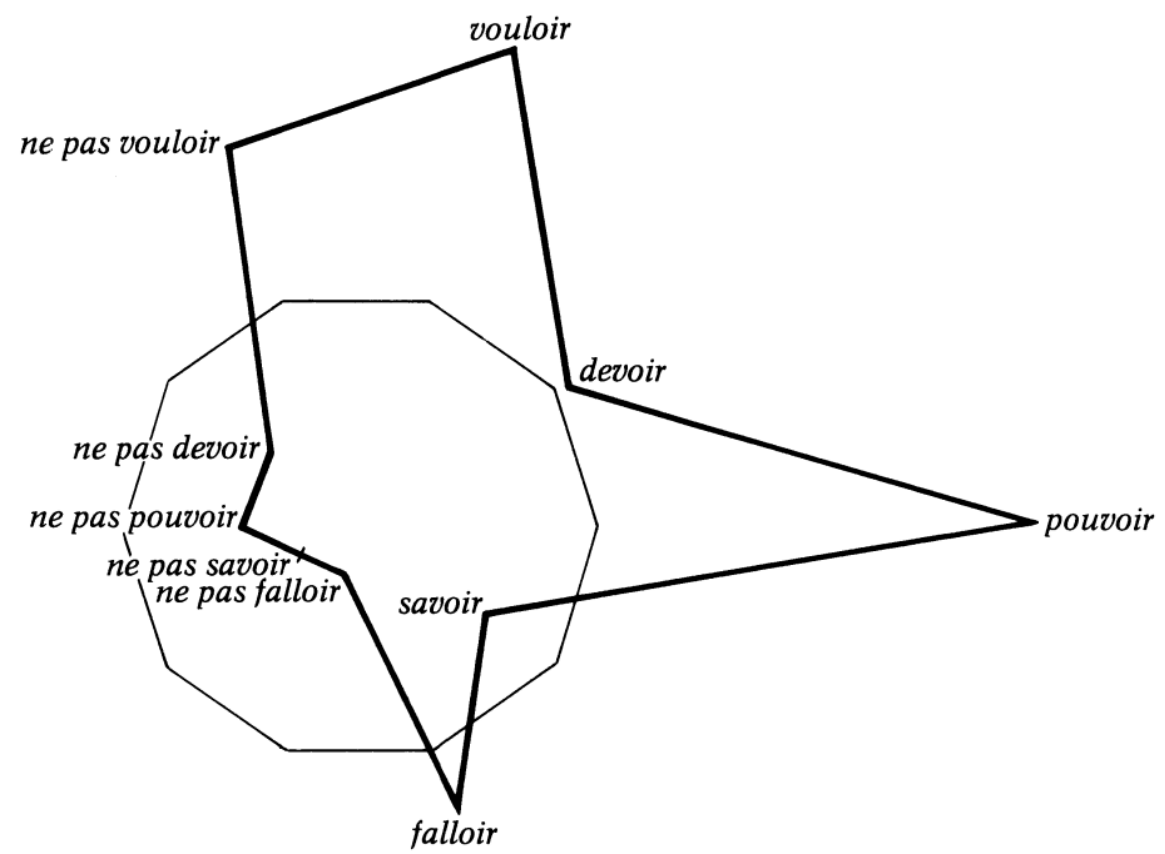


Pour comprendre l'importance de ce phénomène, il faut s'arrêter un instant sur un problème d'interprétation. En effet, le verbe devoir, tout comme pouvoir, et leurs substituts évoqués plus haut, recouvrent deux types d'emplois différents. D'une part, ils peuvent indiquer une obligation ou une capacité juridique : «Le président peut dissoudre quand il le veut» (FM). L'obligation ou la capacité peuvent être morale: «Le peuple doit savoir»(VGE). D'autre part, certains emplois de ces modalités signifient simplement que le locuteur envisage une possibilité, un événement qu'il juge possible, souhaitable. Cela est vrai aussi bien pour pouvoir que pour devoir (ainsi, par exemple, M. Mitterrand à propos des autres énergies «qui doivent nous permettre de diversifier nos sources»). Or près des trois quarts des emplois de devoir dans le discours de M. Giscard d'Estaing sont du type obligation contre un petit tiers chez son concurrent où domine la modalité du possible. De même les emplois de pouvoir par l'ancien président ont pour moitié le sens de permis/interdit, contre seulement un dixième pour M. Mitterrand. Mieux encore, M. Giscard d'Estaing utilise la quasi-totalité de ces permis/interdit dans des énoncés concernant son adversaire. Les lois dégagées par l'analyse sémiotique du récit nous révèlent l'importance de cette constatation :

- «Si le sujet du vouloir faire coïncide avec le sujet de l'énonciation, nous aurons la modalité du vouloir» qui correspond à l'axe «sujet-objet». Coordonnée à pouvoir, elle s'inscrit dans un «faire transformateur qui assure le passage d'un état dans un autre ${ }^{26}$. C'est exactement le propos de M. Mitterrand qui privilégie je veux, je peux avec cette condition d'état suspensive unique, «si je suis élu».

- Au contraire, si «le sujet du vouloir faire est différent du sujet de l'énonciation, nous aurons la modalité du devoir» ${ }^{27}$ dans son acception de capacité ou d'obligation. Telle est bien la caractéristique du discours giscardien modalisé surtout à propos de l'autre et tournant dans ce cas autour de la condition «si vous êtes élu».

Ces emplois très typés s'expliquent par les choix stratégiques des deux candidats que révèle l'analyse de leurs énonciations respectives.

M. Mitterrand a choisi la logique motivante selon laquelle son état de président, à partir du 10 mai, lui décernera compétence pour passer du vouloir faire au pouvoir faire. C'est pourquoi le futur président peut se situer massivement dans l'ordre du discours. A la limite, en dehors des références à la campagne rappelant les programmes et les prises de positions, une seule marque déictique pourrait lui suffire : le 10 mai. Les actants se

\footnotetext{
${ }^{26}$ Courtes (Joseph), Introduction à la sémiotique narrative et discursive, Paris, Hachette, 1980, p. 75.

27 Ibid.
} 
positionnent sans difficulté : vous avant, je après. En utilisant des verbes accomplis au passé pour parler de son adversaire, dont il a l'habileté de sembler laisser à un autre le soin d'instruire le procès, M. Mitterrand peut placer clairement son propos autour d'une tension unique entre lui-même, sa volonté et son auditoire sans avoir besoin, pour atteindre les Français, de recourir à autant de procédés inclusifs que son adversaire.

A l'inverse, M. Giscard d'Estaing a choisi de centrer ses interventions sur l'autre, selon la logique des présuppositions où le «faire transformateur du sujet (ici le vous) implique au préalable une compétence du faire ${ }^{28}$, compétence qu'on tente de lui nier sur tous les plans. C'était évidemment le sens profond du slogan que le candidat sortant avait choisi pour sa campagne. «Il faut un président à la France», présupposait que, contrairement à lui-même, l'autre ne possédait pas le savoir-faire qu'exige préalablement l'exercice du pouvoir. Encore faut-il apporter la preuve de cette incompétence substantielle, donc mettre en scène l'épreuve de réalité grâce à du récit et à de la fiction («si vous êtes élu...»). Bien sûr, l'idéal serait que la preuve vint de la bouche même de l'autre ; alors, on le harcèle de questions sur les communistes, le déficit budgétaire ou même le cours du deutschmark, lors d'un incident qui a beaucoup étonné mais qui se trouve inscrit dans la logique de l'énonciation giscardienne, toute ordonnée par la volonté de déplacer la tension entre le je et le vous- inhérente au choix de départ - vers la relation entre vous et votre vouloir faire (ou être). Ce schéma a été d'ailleurs repris par le président sortant dans sa conclusion à propos du programme économique des socialistes et, surtout, à propos du goût du changement chez les Français. Et si l'on se souvient que le véritable destinataire de l'énoncé n'est pas le contradicteur mais l'auditeur, donc l'électorat, il n'est pas interdit de penser que la principale tension chez $\mathrm{M}$. Giscard d'Estaing réside dans l'opposition entre le risque de ce que les Français pourraient faire le 10 mai et la réalité qui, selon lui, leur dicte d'y renoncer. C'est pourquoi il utilise autant le procédé inclusif non pas seulement pour prendre à témoin mais, bien plus, pour mettre en garde, admonester. C'est la fonction profonde de savoir, surtout lorsqu'on le couple avec falloir («il faut savoir», utilisé plusieurs fois) et avec devoir («vous devez savoir», «les Français doivent savoir», etc.). Ainsi, l'ex-président oppose-t-il au vouloir faire qu'il pressent être, au-delà de son adversaire, le désir de la majorité des électeurs, le principe de réalité et son corollaire, la censure du désir.

Mais le discours du désir ne se trouve pas sans ressource devant une telle censure, comme l'a fort bien montré Freud à propos du mot d'esprit. On pourra en trouver de bonnes

\footnotetext{
${ }^{28}$ Courtès (Joseph), op.cit., p. 76.
} 
illustrations dans les flèches que M. Mitterrand décoche habilement contre son adversaire : «l'homme du passif», «je ne suis pas votre élève», «le ton professoral», «les distinctions sémantiques»... et surtout cette métaphore du conducteur qui a renversé la voiture et demande à l'autre de repasser son permis (il ne sait pas conduire et veut examiner les autres...)

L'ensemble des indices étudiés convergent donc d'une manière presque parfaite, et montrent ainsi combien un tel débat laisse peu de place à l'improvisation et à la spontanéité. Chacun des candidats avait fait un choix et s'y est remarquablement tenu pendant près de deux heures. Cette apparente aisance provient sans doute de ce que les deux stratégies avaient été adoptées depuis longtemps et inspiraient l'ensemble de la campagne présidentielle. C'est en cela que peut résider l'intérêt d'une recherche comme celle-ci car, par ailleurs, il semble illusoire de chercher quel a été le discours le plus «efficace» si tant est que l'on puisse prouver un jour que ce type de confrontation télévisée influence le comportement électoral. 